\title{
Understanding the Dynamics of Change and the Impact on Psychiatric Education
}

\author{
Theodore B. Feldmann
}

Received: 7 March 2014 / Accepted: 17 July 2014 / Published online: 15 August 2014

(C) Academic Psychiatry 2014

\begin{abstract}
Academic departments of psychiatry are experiencing unprecedented changes that are difficult and challenging for faculty and administrators. This article examines the factors that influence change and the barriers to effective change. The author reviewed the business literature on change in organizations and examined the psychodynamic factors that mediate individual and organizational response to change. Several business models for effective change management exist and can be utilized by psychiatric educators. The psychodynamic models of change are useful for understanding the psychological impact of change on organizations and individuals. Effective management of change requires careful attention to the goals of the organization, development of a detailed plan to implement change, adequate resources to carry out the change, effective leadership and communication, and contingency plans for unforeseen events. Individual and organizational needs must also be considered. A model for dealing with change in education is presented.
\end{abstract}

Keywords Administration - Curriculum development . Faculty development

John Kotter, one of the leading authorities on change, writes that, "By any objective measure, the amount of significant, often traumatic, change in organizations has grown tremendously over the past two decades" [1]. Academic departments of psychiatry are clearly experiencing unprecedented changes that are, at best, difficult and challenging for faculty and administrators. These changes include the constantly evolving financial and regulatory landscape of healthcare, new regulations related to residency training and medical student

T. B. Feldmann $(\bowtie)$

University of Louisville School of Medicine, Louisville, KY, USA

e-mail: tbfeld01@louisville.edu education, increased pressure on faculty to generate clinical revenue which decreases time for educational and scholarly activity, a rapidly changing knowledge base that makes it difficult to keep education programs current, and changes resulting from implementation of new technology [2].

The changes impacting psychiatry education programs come from both external and internal sources. External changes are those that originate outside the organization. These include change mandated by the ACGME and LCME, healthcare reform such as the Affordable Care Act, new CPT codes, and the implementation of DSM-5. Internal changes originate from within the organization and include such things as consolidation of hospitals and practice groups, implementation of electronic medical records, and changes in leadership or mission within an institution.

Change is experienced as stressful regardless of its source. There is a commonly held perception, however, that internal change should be easier to deal with than external change. Why is this belief common? Most individuals believe that they will have more input into internal changes than those from external sources [1]. The reality, however, is often very different. External change may actually be easier to accept because it is often mandated by entities over which the individual has little or no control (e.g., the federal government). On the other hand, implementation of internal change without faculty or staff input may lead to feelings of frustration. When people expect to have input into change but the input fails to materialize, they often react with feelings of disappointment, resentment, and even anger.

\section{The Impact of Change}

Change, regardless of the source, has significant impact, both positive and negative, on organizations [3]. When change is positive, the efficiency and productivity of the organization 
are increased, facilitating the achievement of the organization's goals and mission. Individual and group morale are improved and there is an overall sense of cohesion and wellbeing. When change has a negative impact, there is a significant increase in stress, anxiety, and resistance [4]. This, in turn, leads to decreased efficiency and productivity which interferes with achieving the goals and mission of the organization. Another casualty of negative change is common sense - coping strategies or operational plans are adopted that are illogical, poorly conceived, or fail to anticipate unexpected consequences [5]. Morale suffers as a result and feelings of disillusionment and alienation permeate the group. In extreme cases, this leads to increased turnover of personnel which further destabilizes the organization.

When the effect of change on individuals is examined, a similar set of largely negative emotional reactions is observed. These reactions ultimately disrupt the structure and function of the organization [6]. The effects of change on individuals can be summarized as follows. Change creates a general sense of anxiety and stress that may escalate to anger and frustration. Resistance, both active and passive, is understood as a psychological attempt to lessen the intensity of dysphoria; efforts to resist change, however, are usually successful only in the short-term and may, in fact, serve to exacerbate the stress experienced by the organization. Eventually, there occurs a growing sense of withdrawal and isolation within the group. Again, this serves the function of denying the impact of change and temporarily protecting the individual from intense emotional experiences. Suspiciousness also occurs within the organization. The motives for change may be questioned or misinterpreted, resulting in a distrust of leadership and the appearance of "conspiracy theories" that offer incorrect or irrational explanations for change and serve to increase the level of collective unease. Organizational dynamics are disrupted and lead to the emergence of competing factions that attempt to mitigate change or position themselves favorably vis-à-vis the organizational leadership. The resulting power struggles further erode confidence in leadership and increase the anxiety level within the group. As this process escalates, there is an unwillingness to work together which often leads to loss of key personnel. Unforeseen events and unintended consequences further erode confidence in the change process and commitment to its implementation. When these negative effects go unchecked, a gradual but progressive stagnation and disintegration of the organization occurs.

\section{The Psychodynamics of Change}

Change is associated with a number of psychological reactions, most of which are perceived by the individual as negative [7]. These reactions are either dysphoric emotional experiences or disruptions to a person's sense of self [8]. The dysphoric reactions consist of a familiar set of emotions: anxiety, fear, sadness or depression, anger, and frustration. The disruptions to the individual's sense of self may be more subtle and, in many cases, are not readily apparent to others. These inner experiences include loss of self-esteem, identity disturbance, alienation, emptiness, loss of feelings of safety and security, loss of control, helplessness, powerlessness, a diminished sense of competency, loss of autonomy, and loss of mastery [9]. The common theme to disruptions of the self, occurring in response to change, is loss. Change may force people into new roles and new ways of doing things. At the same time, change makes people give up established and valued ways of functioning; for many individuals, their sense of identity is linked to these established behaviors. When change is forced upon an individual or organization, there may not be sufficient time allotted for adjustment to new behaviors or letting go of traditional roles.

Why do people have these intense reactions? To fully understand change, and to deal with it effectively, we must examine why people with apparently healthy personality structures encounter significant difficulties in adapting to new situations. The dysphoric emotional reactions and the loss of self that accompany change are mediated by psychodynamic influences that include unconscious factors, ego strength, the cohesiveness of the self, conflicts, and personality style [10]. These influences operate on both individual and organizational levels. Organizations and leaders must take these factors into account in order to successfully facilitate change.

Diamond has discussed the psychodynamic life of organizations [8]. Organizations tend to be conservative by nature. This philosophy values the status quo and creates an immediate resistance to any type of change. Leaders are likely to view change with skepticism, while members view change with suspicion. The conservative organizational culture has two effects. First, members embrace the conservative organizational philosophy and become resistant to change. When change does occur, it is perceived as excessively traumatic or disruptive. Second, only ambitious and aggressive members advocate change. For these people, change may become a personal crusade. The push for change has an overzealous and unrelenting quality. These people have a significant narcissistic investment in their vision of change. Eventually, the organization is worn down and gives in to change, even when it is detrimental to organizational welfare. This process helps to understand why even bad ideas are accepted.

Two theoretical models are useful in understanding the dynamics of organizational change — object relations theory and self psychology. Both of these approaches draw parallels between organizational dynamics and individual personality development. Dysfunction within organizations can be understood as a breakdown in organizational structure. 
Object Relations Theory

Otto Kernberg described pathological regression in organizations and leaders $[11,12]$. Change may lead to a sense of powerlessness and limited options, experienced at an individual level and by the organization as a whole. This often results in psychologically regressive behavior. Regression, in turn, causes relations between individuals, units, and divisions within the organization to become contentious and riddled with conflict. This regression leads to what Kernberg called "paranoiagenesis in organizations" [12]. He used this term to describe the paranoid-schizoid collapse of individuals in groups and organizations undergoing change.

The object relations approach applies a Kleinian model to dysfunctional group and organizational structures. Change overwhelms systems, leading to dysfunction and defensive regression to a paranoid-schizoid position. The pre-change environment is idealized and viewed as the "good object" and the unstable, change-induced environment becomes the "bad object." Manifestations of this phenomenon include withdrawal, suspicion, anger, acting out, depression, and the use of splitting and projective identification. Again, these reactions occur at both individual and organizational levels.

\section{Self Psychology}

A self psychology understanding of the effects of change on organizations and individuals has been described by several authors [13-15]. This approach is predicated on the work of Heinz Kohut $[16,17]$. Self psychology, like object relations theory, views organizational and individual behaviors as being linked. An organization is a shared environment, and members of the organization have a shared life experience. Out of this shared experience, an organizational self emerges. Shared emotional experiences resonate, resulting in a shared empathic experience - a kinship or twinship among members, resulting in a sense that "we're all in this together."

Disruptions to the shared emotions and experiences, such as change, are experienced by both the organization and its members as unempathic. The unempathic responses may be viewed as coming from the organization itself or from external forces driving change (e.g., government regulations or new educational standards). The perceived unempathic responses result in anxiety, pain, and frustration leading to disintegration anxiety (i.e., disintegration of the organizational self).

When this occurs, the organization and its members seek out people, activities, or situations to restore cohesion. These are organizational selfobjects that provide empathy and cohesion. Examples include charismatic leaders who emerge from within the organization to "save the day," a preoccupation with sameness, or an insistence on adhering to the "old ways" of doing things.
In the absence of organizational selfobjects, maladaptive behaviors emerge that provide temporary, but pathological, structure. These are organizational disintegration products. Examples include resistance to change, reemergence of archaic narcissistic and dependency needs (e.g., a sense of entitlement or the longing for a strong and effective leader), and acting out. The acting out behaviors in organizations include arguments, staff conflict, efforts to sabotage change, passiveaggressive behavior, increased absenteeism, and an increase in grievances or other personnel complaints.

In extreme instances, members will find new selfobjects outside the organization. This results in people leaving for better jobs or devoting increased energy to activities outside the workplace. Certain individuals will display extreme disintegration products which are disruptive to the organization. The organization responds by terminating these persons. The termination is then perceived by the group as unempathic and leads to further fragmentation - thus, a cycle of disintegration ensues.

The self psychology approach to understanding change has the advantage of describing specific steps that can be taken by organizations to prevent the sequence of disintegration and fragmentation that often accompanies change [13]. This is accomplished by planning and implementing change in an empathic manner. In other words, change must be phase appropriate - it must be what the organization needs when the organization needs it. Communication must be open, direct, and truthful. The grandiose and idealization needs of individuals and the organization as a whole should be met. When regressive behavior inevitably occurs, it should be met with calmness and empathic strength, not with punitive or confrontational responses. It should be noted that empathy does not mean doing what people want but, rather, responding to change in a way that recognizes the impact it is having on the members of the organization. The presence of empathic selfobjects and an adequate support system is essential to prevent fragmentation. Finally, change should be implemented at all levels within the organization, not just from the top down. All of these steps facilitate communication, ownership of change, and a sense of cohesion.

\section{Strategies for Managing Change}

Change is inevitable and often accompanied by intense reactions. An important question to consider is whether change must always be difficult and painful. More importantly, are there ways to make change less difficult? Four business models for dealing with change will be examined. Elements common to all successful models are identified and recommendations for dealing with change in education will be made. 


\section{Kotter's Model for Leading and Implementing Change}

The first model for change comes from John Kotter [1]. This model emphasizes the importance of planning for change and the need for a sequential implementation process. Kotter identifies eight stages, the first of which is establishing a sense of urgency (i.e., why change is necessary and must be done now). The organization must then create a guiding coalition to lead the group through the change process. This coalition develops a vision and strategy for change. Specifically, what does the organization want to become, how is the goal attained, and what resources are required for successful implementation? The next phase involves communicating the change vision to everyone in the organization. The communication phase may be the most important because without it, the organization will not fully comprehend the rationale for change. Leaders must also empower broad-based action in order to fully engage the organization and create a sense of ownership within the group. Kotter describes change as a long-term process often taking years to achieve. During extended implementation phases, it is important for the organization to see tangible progress; this is what Kotter means by generating short-term wins. As short-term wins occur, they are accompanied by new energy for further change. Kotter describes this as consolidating gains and producing more change. Finally, the changes must be incorporated into the culture of the organization. There must be a sense of "this is how we do things here." Kotter calls this phase anchoring new approaches in the culture of the organization.

\section{Kanter's Model of Organizational Change}

Another model of change was proposed by Rosabeth Kanter [18]. Her model focuses on organizational factors. The first step for Kanter is to analyze the organization and the need for change. Once this assessment has been made, the organization must create a vision and a common direction. Again, the crucial question is - what do we want the organization to look like in the future? Change often involves letting go of old ways of doing things in favor of new, and, presumably, better methods. For this to occur, the organization must separate from the past. Kanter believes that a sense of urgency must be created that facilitates letting go of traditional roles or procedures. The support of a strong leader is also necessary for change to succeed. The leader must share the vision for change and reinforce the sense of urgency that change needs to occur now. Support from the leader alone, however, may be insufficient to guarantee success. Every organization is composed of factions whose support is essential for meaningful change to occur. Thus, Kanter recognizes the importance of lining up political sponsorship. In the end, even the best ideas fail without an adequate implementation plan. A related essential component is the development of enabling structures, which represent the resources necessary for change to succeed. As the change process unfolds, it is necessary to communicate, involve people, and be honest. These steps engage the members of the organization and facilitate a sense of ownership of the change. A foundation is laid that reinforces and institutionalizes change.

\section{Luecke's Model of Teamwork and Change}

Richard Luecke describes a model of change that emphasizes teamwork and organizational issues [19]. The change process begins by mobilizing energy and commitment through joint identification of problems and their solutions. Once problems have been identified, the organization develops a shared vision of how to manage change. Identification of leadership allows the organization to begin to move forward with change. Throughout the process, it is important to focus on results, not activities - a results-driven approach enables the organization to move forward with meaningful change and avoid being caught up in "going through the motions" of change. Leucke believes it is essential to begin change at the periphery of the organization and then let it spread to other units. This approach avoids the pitfall of pushing change from the top down and facilitates a sense of ownership within the group. As change proceeds, it must be institutionalized. The change process must also be monitored for problems and unintended consequences and adjusted to maximize a positive outcome.

\section{Bridges' Model of Transitions and Change}

The final business model for change was articulated by William Bridges [3]. This model emphasizes the importance of transitions and psychological factors [20]. Bridges believed that change involves a series of transitions in which people adjust to and accept new roles as they abandon previous ways of doing things. The first stage of change is the endings phase. Members of the organization recognize that change is necessary and that roles will be redefined. This requires terminating, or saying goodbye, to traditional ways of operating. Bridges believes that an organization cannot move forward until it successfully lets go of the past. Once this occurs, the organization enters into a neutral zone in which new tasks and structures are introduced. This is the phase during which the planned change is actually implemented. When change has occurred, the organization is ready for a period of beginnings when members embrace the change and come to identify with their new roles and responsibilities.

\section{The Common Characteristics of Successful Change}

In spite of unique terminology and sequencing, each of the change models described in this section shares many 
characteristics. Examination of these models allows for the identification of essential elements for successful change. First, a vision, or strategic plan, is created for the organization. All change efforts are directed toward carrying out the vision and communicating its importance to everyone in the organization. Leadership must create a team to bring about change. The team engages in detailed planning for change. The plan must then be implemented in a stepwise manner. The organization must have adequate resources to support change. Again, all levels of the organization must be involved in planning and implementation to maximize success. It is critical to maintain open communication and input throughout the process. A feedback loop is necessary to monitor progress along with contingency plans to deal with adverse events. Finally, anticipation of unforeseen events and unintended consequences is essential; failure to plan for complications can undermine the change process.

\section{Why Is Change So Difficult?}

People resist change for a variety of reasons. Loss of control, interfering with a person's sense of autonomy and self-determination, is a frequent theme [18]. Change is always accompanied by uncertainty; when the level of uncertainty is excessive, people will resist the unknown. Unexpected or disruptive decisions imposed on people without notice or input will be rejected. Another common experience during times of change is that everything feels different; members of an organization become accustomed to doing things in a certain way. Change disrupts that routine [21]. For these reasons, when major change is implemented, it should be done in a stepwise manner to allow people to adjust to new ways of doing things. For many in organizations, their sense of identity and purpose is linked to what they do. When change occurs abruptly, that sense of identity is temporarily lost, resulting in emotional discomfort that leads to resistance of the change [18]. Concerns about competence also occur when new approaches are radically different and lead to fears that change cannot be mastered [9]. Change frequently requires an increase in workload, particularly during the early phases. If the workforce is already overburdened, then the change will be rejected even if it ultimately leads to increased productivity and efficiency. Past resentments may resurface or become intensified during times of change [18]. Healing gestures are often necessary before change can proceed. Finally, it must be recognized that change, even under the best of circumstances, can be painful and intensify feelings of loss. Change sets in motion a ripple effect-it does not occur in isolation and the effects of change must be examined throughout the organization to minimize unintended consequences. Resistance to change is not necessarily pathological; it should be expected and planned for.

\section{Why Do Change Efforts Fail?}

The failure rate of change is approximately $70 \%$ [22]. The failure rate is due, in part, to the unique dynamics of change. The need for change is unpredictable, resulting in change efforts that are either poorly conceived or triggered by a crisis for which the organization is illprepared. In these cases, planning and implementation are inadequate, leading to situations in which change is destined to fail.

Multiple factors contribute to the failure of change [23, 24]. Complacency is among the most common. Organizations become too comfortable with the way in which they operate and grow too confident of their ability to adapt to changing circumstances. Complacency acts in opposition to the planning and sense of urgency necessary for a successful outcome [23]. The failure to create a guiding coalition is another factor in unsuccessful change. Without this coalition, there is no one to navigate the organization through the change process. Lack of vision and inadequate planning contribute to failure because the organization is unsure of what to do or how to do it. Poor communication undermines change efforts because members of the organization fail to appreciate the importance of and rationale for change. Failure to anticipate and deal with unexpected obstacles further decreases the likelihood of success. A common example of such an obstacle is the lack of adequate resources to successfully implement change; the vision and implementation plan may be adequate but failure occurs because the organization lacks the resources or infrastructure to complete the process. Failure to achieve short-term, observable results is another factor that interferes with successful change; the organization must see tangible results in a relatively short period of time in order to maintain the energy for sustained change. A related problem is ending the change process too soon. This occurs when leadership misinterprets shortterm results as evidence of lasting change. In these instances, the change process is terminated prematurely before the desired outcome is obtained. Finally, change will fail unless it is firmly anchored in the organization's culture.

To summarize, change fails (1) when there is poor conceptualization of what the organization wants to accomplish, (2) without a clearly articulated implementation plan that utilizes common sense and understands the unique dynamics of the organization, (3) in the absence of adequate and effective leadership, (4) without adequate communication, (5) without the empowerment and engagement of those affected and a sense of group and individual 
ownership, (6) when adequate resources to achieve goals are unavailable, and (7) without involvement of all levels of the organization.

Unsuccessful or failed change has consequences and often results in significant organizational backlash. Future attempts at change will be viewed with skepticism and are much more likely to fail. Trust in leadership erodes following failed change efforts. Once this occurs, it may be impossible to regain momentum for change. A long history of multiple failed change efforts makes it difficult, if not impossible, to create change without a massive organizational restructuring and change in leadership.

\section{The Importance of Leadership in Guiding Change}

Organizations cannot function effectively without adequate leadership [25]. By extension, meaningful change will not occur without strong leadership. The connection between leadership and change is succinctly described by Warren Bennis - "A leader is, by definition, an innovator. He does things other people haven't done or don't do. He does things in advance of other people. He makes new things. He makes old things new" [26].

Leadership and management are not the same thing [1]. This distinction is important when considering the effect of change on organizations. Leadership sets the vision for the organization, while management carries out the tasks that achieve the vision. Leadership establishes the direction of the organization and defines its vision for the future. The leader then aligns people through communication and the creation of teams and coalitions. Leaders provide the motivation and inspiration to overcome barriers to change. Management, on the other hand, implements the vision and direction identified by the organization's leadership. They oversee planning and budgeting to achieve results through the proper allocation of resources. Management is also responsible for organizing and staffing to provide the structure necessary for implementing change. Finally, management exercises a controlling and problem-solving function to overcome barriers to change. A delicate interaction between leadership and management is required for successful change yet the boundaries between these two components of the organization must remain intact.

The qualities of effective leaders include honesty, integrity, and fairness. Leaders must possess a strong conviction that change is needed and believe in the outcomes of that change. It is rare, however, that a leader will have all the answers to an organization's needs. The successful leader is open to input from others while maintaining the overriding vision for the organization [26]. Effective leaders are willing to share the work and share in the sacrifices needed for success. The leader who mandates change and then sits idly while others carry out the tasks will quickly lose respect and will likely fail. Successful leaders are also willing to give credit to others and share the rewards of the group's accomplishments. In other words, effective leaders recognize that, no matter how strong their vision, change cannot be driven by the force of their will alone. Change only occurs when the vision is coupled with teamwork, cooperation, and a sense of ownership throughout the organization.

The importance of leadership in change can be summarized as follows. Effective leaders have a vision for the organization. They create an environment in which ideas and creativity are encouraged. Leaders assemble a team to carry out the vision. They understand the importance of planning and preparation. Throughout the change process, leadership communicates the need for change. They understand and anticipate the reaction of group members to change and take steps to assist the organization in making the necessary adjustments. Effective leaders also recognize that change often does not go as planned. They are willing to modify and adapt plans to meet the needs of the organization and its members.

The unique structure of academic departments must be taken into consideration when discussing the roles of leadership and management. Large departments may have complex administrative structures in which the roles of leadership (e.g., the chair and vice chairs) are clearly delineated from management (e.g., service chiefs and program directors). In smaller departments, there may be significant overlap between leadership and management. The principles of change management can be utilized by departments of all sizes but may need to be modified to fit the unique needs of a specific institution.

\section{Managing Change in Psychiatric Education}

What is the relevance of the preceding discussion for psychiatric educators? Consider the following scenario. A training director or director of medical student education is asked to create or modify a course, clinical rotation, or curriculum. Where do you start? How do you accomplish the change? The previous discussions demonstrate that how change is approached impacts the outcome. How change is implemented is crucial to success or failure. The concepts illustrated above can be applied to managing change in psychiatric education.

One of the earliest steps in managing change in psychiatric education is identifying or creating an effective leadership structure. Most academic departments of psychiatry have an existing organizational structure for education. The chair designates a vice or associate chair for education whose role is to set the overall educational philosophy for the department. The 
vice/associate chair for education also leads in the implementation of educational strategies adopted by the department or put forward by the dean. Each educational component of the department also has its own leadership-residency training director, director of medical student education, and fellowship directors. These individuals work closely with the vice/associate chair for education and should, ideally, collaborate to set the educational priorities of the department. Faculty should be identified to serve as education coordinators at each training site; these coordinators are responsible for making sure that the curriculum is adequately carried out at each training site.

The essential elements for dealing with change in psychiatric education are similar to those identified earlier in this paper. Educational leadership establishes the department's vision for the future. This vision describes what the department's educational programs will look like. Next an implementation plan is developed. The central question here is how to put the department's vision for education into practice. The vision and the implementation plan are then communicated to everyone in the department. The emphasis during this phase is on engaging all members of the department in the change process and creating a sense of ownership in which the need for change is accepted and valued. A key step in creating the sense of ownership is an understanding of the psychodynamic effects of change on both individuals and systems. Finally, adequate resources must be available to successfully bring about change.

Table 1 describes the activities necessary to facilitate change in psychiatric education. It is necessary to create an education infrastructure which includes a forum for sharing ideas and planning for change. The establishment of an education council or advisory group, which meets regularly and is composed of the leaders of all education programs, will assist

Table 1 Facilitating change in psychiatric education

- Establish an education infrastructure which includes a forum for sharing ideas and planning for change

- Create your own vision and mission statements and align these with the department and university

- Develop a strategic plan for education

- Utilize change as a catalyst - anticipate change

- Be proactive - remember, reactive change tends to fail

- Create an organizational structure at the unit level

- Establish a support system for faculty and trainees

- Emphasize faculty development

- Utilize and embrace technology (but do not overextend your resources)

- Identify and allocate the resources necessary for change

- Focus on the individual (e.g., faculty morale and individual needs to avoid organizational fragmentation)

- Keep change in perspective (remember what you are working toward)keep it simple and realistic the department in planning and implementing change. One of the functions of the group is to create a strategic plan for education; this involves articulating the department's mission statement for education and aligning those educational goals and objectives with broader department and university goals. The strategic plan for education focuses on curriculum development and implementation as well as planning for the future. This process asks the question, "Where are we now and where do we want to go with our education programs?" It is also important to utilize change as a catalyst; anticipate change and use it as an opportunity for further growth and development. Educators should be proactive in dealing with change; have ideas and plans in place before a crisis occurs or massive change is mandated by external sources, keeping in mind that reactive change tends to fail. Create an organizational structure at the unit level to deal with education issues; this fosters communication between program coordinators and faculty/ supervisors at each training site. Because change can be stressful, it is useful to establish a support system for faculty and trainees. This support system should emphasize faculty development. Utilize and embrace technology to the extent that departmental resources allow [27]. While technology is useful, it must be remembered that technology is also expensive and resources must not be overextended. Identification and allocation of resources necessary for change is an important part of the education infrastructure. These decisions may need to be made after consultation with the chair but should always involve senior educators. Finally, successful change must focus on the individual-faculty morale and individual needs should be addressed to avoid undue stress and burnout, facilitate buy-in to change, and avoid organizational fragmentation. This also helps to keep change in perspective and maintain focus on educational and organizational goals.

\section{Summary}

Change can be difficult and unpleasant. But, change is inevitable. In academic psychiatry, change often feels unrelenting. The more intense and persistent the change, the more likely it is to be resisted. Dynamics unique to every organization and its members may make the change process more difficult. One thing is clear, change efforts will fail without adequate planning and nurturing. Change will be easier on the organization and will be more likely to succeed with attention to certain details. It is important that an organization utilize its strengths and existing resources to facilitate change. It may be easier to build on existing structures rather than dismantling existing programs and starting over. While the magnitude of change may make it tempting to "wipe the slate clean and start over," few 
departments have the financial or personnel resources to undertake such a rebuilding process. In the end, planning and communication are essential for making change work.

Implications for Educators

- Change has the potential to negatively impact psychiatric education programs.

- It is important for educators to be aware of the various models for managing change as well as the institutional and interpersonal factors that mediate change.

- A clear vision for education programs must be developed along with specific educational goals.

- Planning and implementation strategies for change must be carefully developed and effectively communicated.

- Psychiatric educators should create an infrastructure within their department to deal with change.

Implications for Academic Leaders

- Change is inherently difficult for organizational and academic systems.

- Academic leaders and their departments are currently experiencing unprecedented levels of change.

- Detailed planning and implementation strategies are essential for successful change.

- It is important for leaders to clearly communicate the rationale for change and to involve all members of the organization in the change process.

- Academic leaders must understand the complex emotional and psychological reactions to change in order to minimize disruption to the organization.

Disclosures The corresponding author states that there is no conflict of interest.

\section{References}

1. Kotter JP. Leading change. Boston: Harvard Business Review Press; 2012.

2. Yager J. The practice of psychiatry in the 21 st century: challenges for psychiatric education. Acad Psychiatry. 2011;35(5):283-92.

3. Bridges W. Managing transitions: making the most of change. Cambridge, MA: Perseus Publishing; 2003.
4. Bovey W, Hede A. Resistance to organisational change: the role of defence mechanisms. J Manag Psychol. 2001;16(7):534-48.

5. Wittig C. Employees' reactions to organizational change. OD Pract. 2012;44(2):23-8.

6. Bordia P, Hobman E, Jones E, Gallois C, Callan V. Uncertainty during organizational change: types, consequences, and management strategies. J Bus Psychol. 2004;18(4):507-32.

7. Campbell RJ. Change management in health care. Health Care Manag. 2008;27(1):23-39.

8. Diamond MA. The unconscious life of organizations. Westport, CT: Quorum Press; 1993.

9. Eilam G, Shamir B. Organizational change and self-concept threats: a theoretical perspective and a case study. J Appl Behav Scie. 2005;41(4):399-421.

10. Diamond M, Allcorn S. The cornerstone of psychoanalytic organizational analysis: psychological reality, transference and countertransference in the workplace. Hum Relat. 2003;56(4): 491-514.

11. Kernberg O. Regression in organizational leadership. Psychiatry. 1979;42(2):24-39.

12. Kernberg O. Ideology, conflict, and leadership in groups and organizations. New Haven, CT: Yale University Press; 1998.

13. Stein HF. Culture change, symbolic object loss, and restitutional process. Psychoanal Contemp Thought. 1985;8:301-32.

14. Muhlbacher J. Role models in leadership and self psychology according to Kohut. J Glob Bus Technol. 2009;5(2):32-9.

15. De Vries MK, Engellau E. A clinical approach to the dynamics of leadership and executive transformation. In: Nohria N, Khurana R, editors. Handbook of leadership theory and practice. Boston: Harvard Business School Press; 2010.

16. Kohut $\mathrm{H}$. The analysis of the self. New York: International Universities Press; 1971.

17. Kohut H. The restoration of the self. New York: International Universities Press; 1977.

18. Kanter RM, Stein BA, Jick TD. The challenge of organizational change. New York: The Free Press; 1992.

19. Luecke R. Managing change and transition. Boston: Harvard Business School Press; 2003.

20. Elrod PD, Tippett DD. The "death valley" of change. J Organ Chang Manag. 2002;3:273-91.

21. Beer M, Nohria N. Cracking the code of change. Harv Bus Rev. 2000;78(3):133-41.

22. Balogun J, Hope Hailey V. Exploring strategic change. London: Prentice-Hall; 2004.

23. Kotter JP. A sense of urgency. Boston: Harvard Business School Press; 2008.

24. Kanter RM. Change masters. New York: Simon and Schuster; 1984.

25. Greiner CB. Leadership for psychiatrists. Acad Psychiatry. 2006;30(4):283-8.

26. Bennis W. On becoming a leader. Reading, PA: Perseus Press; 1994.

27. Hilty DM. The present and future interface of technology and medical education. Acad Psychiatry. 2006;30(6):437-8. 